\title{
Influence of Electric Fields on the Flow of a Liquid Crystal Mixture in Circular-Pipe Electrodes*
}

\author{
Tetsuhiro TSUKIJI ${ }^{* *}$, Eitaro KOYABU**, \\ Tomohiro TSUJ' ${ }^{* * *}$ and Shigeomi $\mathrm{CHONO}^{* * *}$
}

\begin{abstract}
Two types of circular-pipe electrode are designed to control the pressure and flow rate of electrorheological(ER) fluids under the application of an electric field. The shape of the electrode is a circular pipe and some parts of the inner surface of the pipe are made of electrode strips. A liquid crystal mixture is selected as a homogeneous ER fluid and the pressure drop in the circular-pipe electrode is measured at constant flow rates. On the other hand, numerical analysis of the electric field and the fluid flow in the circular-pipe electrode is conducted. It is assumed that the viscosity, which depends on the electric field intensity, is distributed in the flow fields. The relationships between the flow rate and the pressure are simulated numerically for various electric field intensities, which agree with experimental results. The difference in the ER effect between the two types of electrodes is discussed on the basis of the distributions of the electric field intensity and the pressure drop. Furthermore, the influence of both the number of electrode strips and the gaps between electrode strips in the pipe on the flow rate vs. pressure characteristics is investigated numerically, and a comparison of the flow characteristics between the present electrodes and two types of parallel-plate electrodes is conducted.
\end{abstract}

Key Words: Functional Fluid, Liquid Crystal, Numerical Analysis, ER Fluid, Flow Measurements

\section{Introduction}

Fluids whose rheological properties such as apparent viscosity can be controlled using electric or magnetic fields from the outside are known to be one of the functional fluids nowadays. In particular, a fluid whose properties can be controlled using electric fields is said to be an electrorheological(ER) fluid. Many research works on welfare devices, micromachines and other applications of ER fluids have been carried out. Liquid crystals are classified under homogeneous ER fluids without precipitation and therefore exhibit high reliability. The areas of application of liquid crystals are expanding and many more applications are expected in the future.

In our study, the case for the pressure mode is consid-

* Received 20th December, 2004 (No. 04-4256)

** Department of Mechanical Engineering, Sophia University, 7-1 Kioi-cho, Chiyoda-ku, Tokyo 102-8554, Japan. E-mail: t-tukiji@sophia.ac.jp

*** Department of Intelligent Mechanical Systems Engineering, Kochi University of Technology, Tosayamada-cho, Kochi 782-8502, Japan ered, which involves controlling the pressure or the flow rate of the flowing liquid crystal using the electric field. Electrodes are necessary to apply the electric field on the ER fluid in this case. Many types of electrodes have been designed to control the apparent viscosity of the ER fluids. In a previous study of the pressure mode, the typical electrodes were a two-parallel-plates electrode ${ }^{(1)-(4)}$ and a two-concentric-cylinders electrode ${ }^{(5)}$, which were applied as an ER valve. Recently, one-sided pattern electrodes were developed $^{(6)}$ and the ER effects were investigated. A one-sided pattern electrode is an electrode in the form of a pattern arranged on only one of the two surfaces of a pair of disks, with an insulator arranged on the other surface. The large length of the electrode perpendicular to the flow direction is needed to obtain a high intensity in the uniform electric field between the electrodes and to avoid a nonuniform intensity near the electrode edge for the twoparallel-plates electrode and one-sided-pattern electrode. It is difficult to connect the two-parallel-plates electrode with a circular pipe when the liquid crystal flows through the circular pipe and the two-parallel-plates electrode because of the different shapes of the cross sections of the 
two flow channels. For the two-concentric-cylinders electrode, the configuration within the electrode for mechanically supporting the inner cylinder is complex.

For the above reasons, circular pipes with the electrode strips on some parts of the inner surface of the pipe were developed $^{(7),(8)}$ by one of the authors. The diameter of the pipe is $1 \mathrm{~mm}$ and the length is $10 \mathrm{~mm}$. The advantage of our electrode is that a flexible tube electrode can be designed easily on the basis of the results of our study because the cross-sectional shape of the circular-pipe electrode perpendicular to the axis is circular. Moreover, a thick electrode with a long diameter can be developed by combining many circular-pipe electrodes and the flow rate can be increased. The flexible electrode can not be designed using the two-parallel-plates electrode and the twoconcentric-cylinders electrode.

In this study, a circular pipe with one pair of electrode strips is designed in order to investigate the influence of the number of electrode strips on the flow characteristics under the application of electric fields, by comparing the results for the pipe with one pair of electrode strips with those for the circular pipe with four pairs of electrode strips developed in our previous research ${ }^{(7),(8)}$. The electrode strips are set up in the flow direction so that the director of the liquid crystal is perpendicular to the flow direction near the electrode strips under application of the electric fields. The voltages are applied in the peripheral direction. Liquid crystals are a kind of homogeneous ER fluid. A liquid crystal mixture ${ }^{(2),(7),(8)}$ composed of some kind of nematic liquid crystal is used to obtain a strong ER effect. Pressure drops are measured using pressure transducers at constant flow rates when voltages are applied. The open-loop test facility with the liquid crystal is set in a pyrostat to keep the temperature constant. Using the theoretical approach developed in this research, the numerical analysis of both the electric fields and the flow in the circular-pipe electrode is conducted and the relationship between the flow rate and the pressure are obtained numerically for various electric field intensities, which almost agree with the experimental results for both circularpipe electrodes. In our numerical analysis, it is assumed that the viscosity of the liquid crystal mixture is distributed throughout the flow field. In our previous study ${ }^{(8)}$ the pressure drop could not be estimated under the application of a high voltage because average viscosity was used without the distribution of the viscosity. The ER effect of two circular-pipe electrodes is discussed from the present calculated and experimental results. Furthermore, using the present calculation method, the effects of both the number of electrode strips and the gaps between electrode strips on the pressure vs. flow rate characteristics are investigated, and the characteristics of the present electrodes are compared with those of the two-parallel-plates electrode in order to design the circular pipe electrode or the two parallel-plates electrode according to their purpose.

\section{Experimental Setup and Methods}

The circular pipes used in the present experiment with the electrode strips on some parts of the inner surface of the pipe are shown in Figs. 1 and 2. The configurations with one pair and four pairs of electrode strips are used to obtain the large flow resistance under the application of the electric fields. The electrode strips are set up in the flow direction so that the director of the liquid crystal is perpendicular to the flow direction near the electrode strips under application of the electric fields. The voltages are applied in the peripheral direction. The length of the circular pipe with electrode strips is $10 \mathrm{~mm}$ and the internal diameter is $1 \mathrm{~mm}$. The four pairs of electrode strips on the inner surface of the pipe are designed as shown in Fig. 1 and the pipe with one pair of electrode strips is shown in Fig. 2. The gap in the peripheral direction between the electrode strips is $0.2 \mathrm{~mm}$ for both electrodes and the total area of the electrode strips for electrode B is larger than that of electrode A. The liquid crystal mixture is used as one of the homogeneous ER fluids. This is the material that is mixed with some kinds of nematic liquid crystals, and its viscosity is higher than that of the nematic liquid crystal.

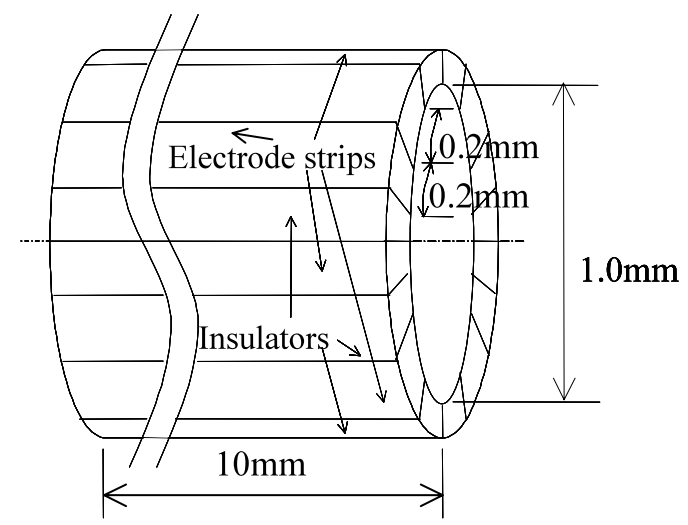

Fig. 1 Electrode A

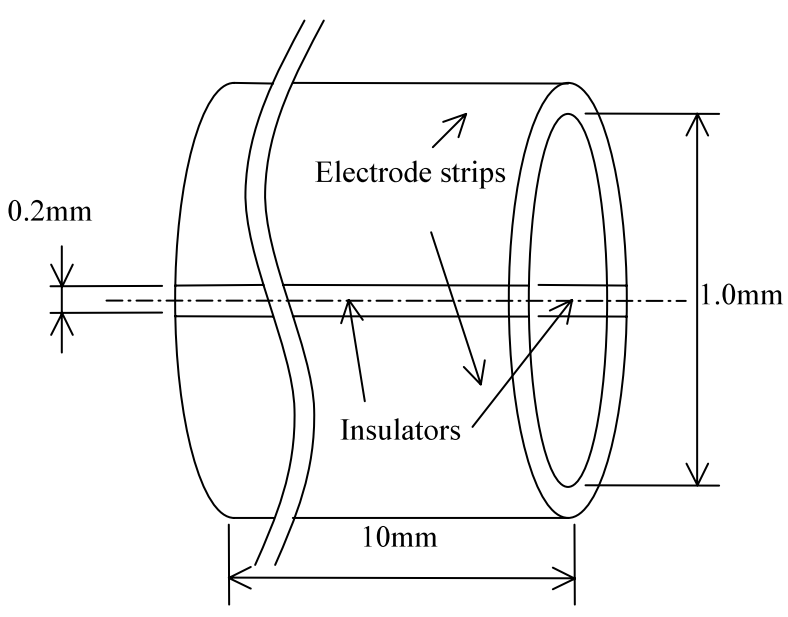

Fig. 2 Electrode B 


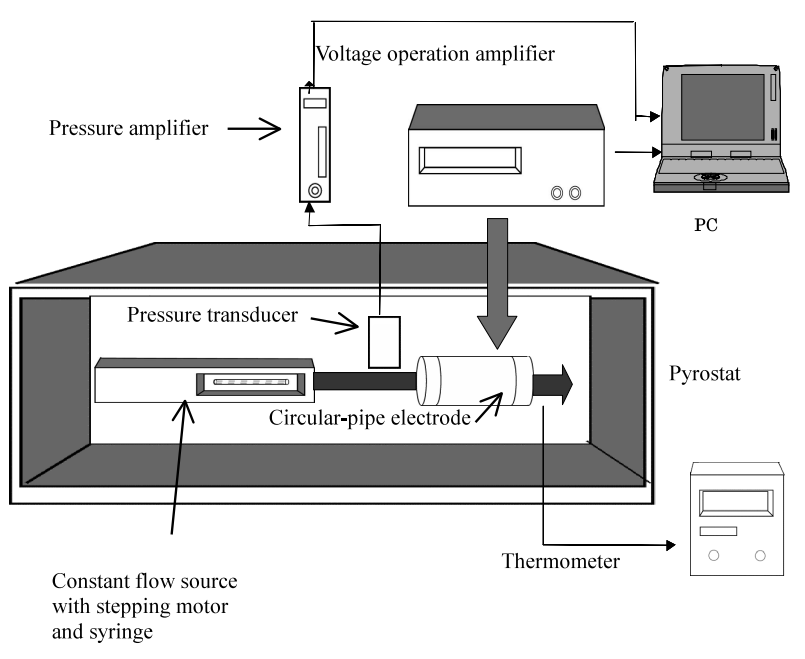

Fig. 3 Experimental apparatus

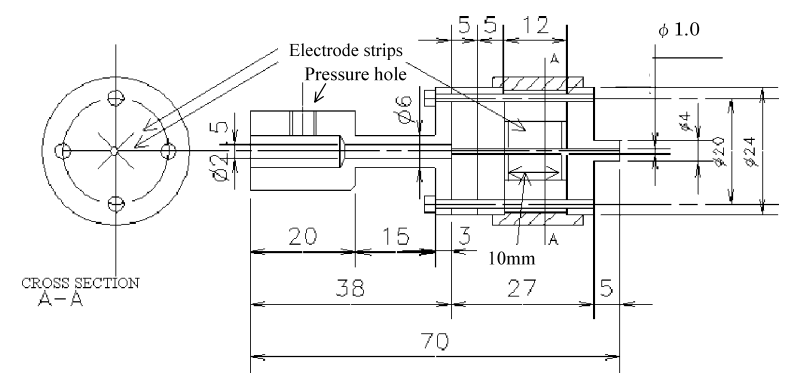

Fig. 4 Flow channel for electrode A

The liquid crystal mixture was supplied by MERCK Japan Co., Ltd. It has a positive dielectric constant. The isotropic nematic transition is at $90.0^{\circ} \mathrm{C}$ and smectic-nematic transition is at $-44.0^{\circ} \mathrm{C}$.

The present experimental setup is shown in Fig. 3 . The liquid crystal mixture is supplied to the circular-pipe electrode at the constant flow rates from the syringe whose piston is pushed by a stepping motor at a constant speed. The flow rates can be controlled by the speed of the stepping motor. The syringe is connected to the electrodes using a glass tube. The inlet pressure of the circular-pipe electrode is measured using the pressure transducer. The flow circuit with the syringe, the glass tube and the electrodes is set in the pyrostat to keep the temperature constant $\left(25 \pm 0.5^{\circ} \mathrm{C}\right)$. The error of the pressure measured in the present experiments is $0.02 \mathrm{kPa}$. The detailed figure for electrode A is shown in Fig. 4. In the case of electrode $\mathrm{B}$, the part of the flow channel with the electrode strips in Fig. 4 is replaced for that of the flow channel of electrode B. The outlet pressure is atmospheric.

\section{Numerical Analysis}

The cross section of electrode A is shown in Fig. 5 . The distribution of the voltage is calculated numerically using the finite difference method by solving the following Laplace's equation, assuming that the voltage is constant in the axial direction. The spacing of the finite difference

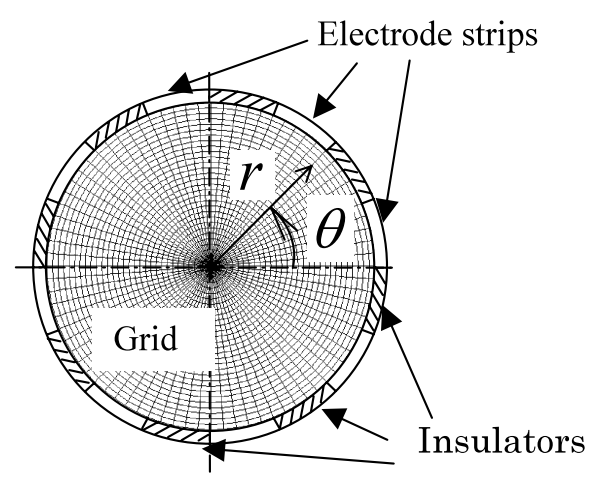

Fig. 5 Cross section of electrode A

mesh is $0.017 \mathrm{~mm}$ in the radial direction and the angle between two mesh points in the circumferential direction is 4.5 degrees, as shown in Fig. 5. The angle is 0.9 degrees with same spacing when the gap is $0.04 \mathrm{~mm}$. The distribution of the voltages can be obtained for electrode B using the same method.

$$
r^{2} \frac{\partial^{2} V}{\partial r^{2}}+\frac{\partial^{2} V}{\partial \theta^{2}}+r \frac{\partial V}{\partial r}=0
$$

where $V$ is the voltage, $r$ and $\theta$ are the radial and the peripheral coordinates.

The electric field intensity $E$ is defined by the following equation.

$$
E=\sqrt{\left(\frac{\partial V}{\partial r}\right)^{2}+\left(\frac{1}{r} \frac{\partial V}{\partial \theta}\right)^{2}}
$$

In the present calculation, the viscosity is assumed to be distributed in the flow field according to the distribution of the electric field intensity. Supposing that $v_{r}=0$ and $v_{\theta}=0$, $\partial v_{z} / \partial z=0$ is obtained from the continuity equation, where $v_{r}, v_{\theta}$ and $v_{z}$ are the velocity components in the $r, \theta$ and $z$ directions, respectively.

From the Navier-Stokes equations in the $r$ and $\theta$ directions, $\partial p / \partial r=0$ and $\partial p / \partial \theta=0$, where $p$ is the pressure. From the Navier-Stokes equation in the axial direction, the following equation is obtained assuming the same assumptions mentioned above.

$$
\begin{gathered}
-\frac{\partial p}{\partial z}+\mu\left(\frac{1}{r} \frac{\partial v_{z}}{\partial r}+\frac{\partial^{2} v_{z}}{\partial r^{2}}+\frac{1}{r^{2}} \frac{\partial^{2} v_{z}}{\partial \theta^{2}}\right) \\
+\frac{\partial \mu}{\partial r} \frac{\partial v_{z}}{\partial r}+\frac{1}{r^{2}} \frac{\partial \mu}{\partial \theta} \frac{\partial v_{z}}{\partial \theta}=0
\end{gathered}
$$

where $\mu$ is the viscosity. After $\partial p / \partial z$ and $\mu(r, \theta)$ are given by the experimental conditions and the calculated results, the axial velocity component $v_{z}$ can be calculated using Eq. (3) by the finite difference method and the flow rate can be also obtained by integrating the axial velocity component. In the present study, the relationship between the viscosity and the electric field intensity is given by

$$
\mu=0.0303+0.0272 E
$$

where the dimensions of $\mu$ and $E$ are $\mathrm{Pa} \cdot \mathrm{s}$ and $\mathrm{kV} / \mathrm{mm}$, respectively. The above equation was obtained by the experimental results for two-parallel-plates electrode at $25^{\circ} \mathrm{C}$ 
$V[\mathrm{~V}]$

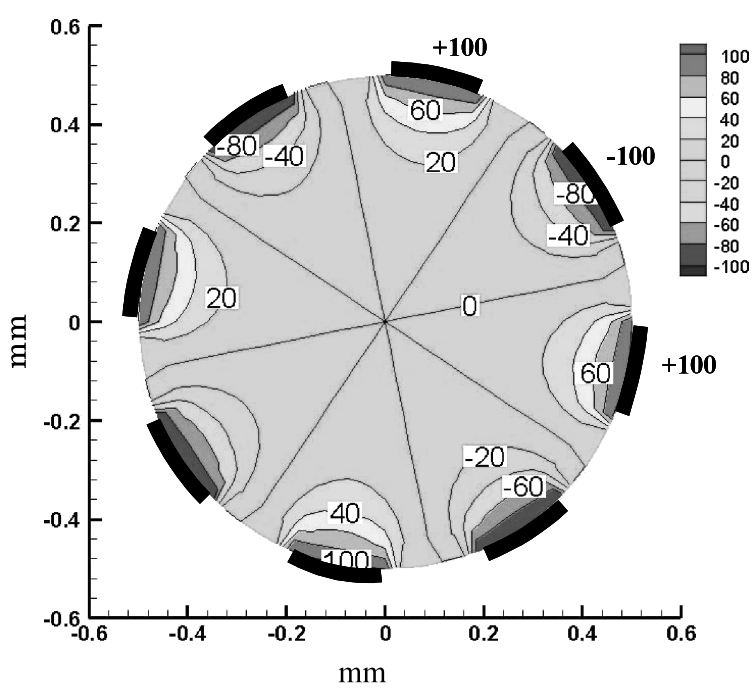

(a) Voltage

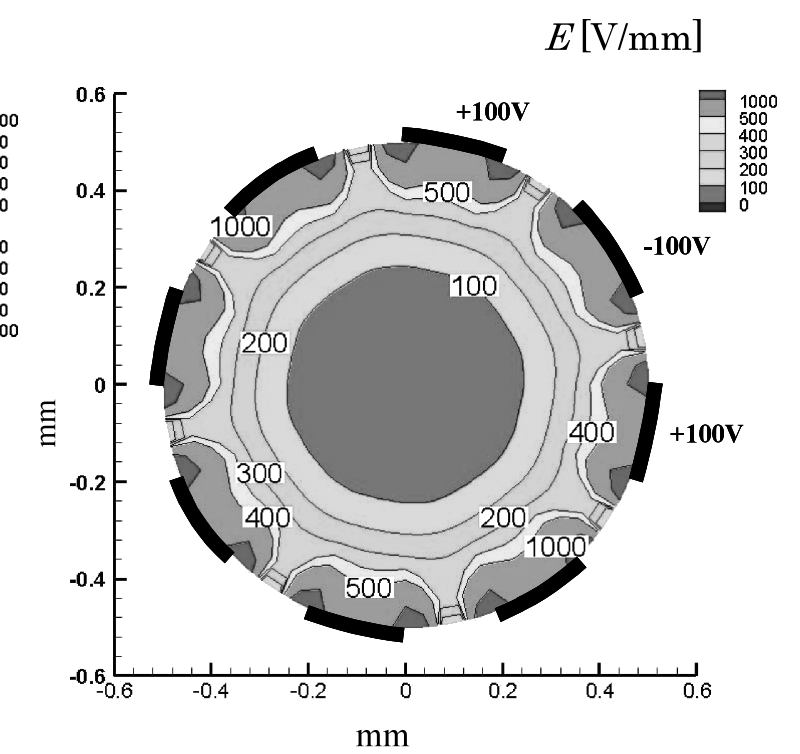

(b) Electric field intensity

Fig. 6 Electric field analysis for electrode A

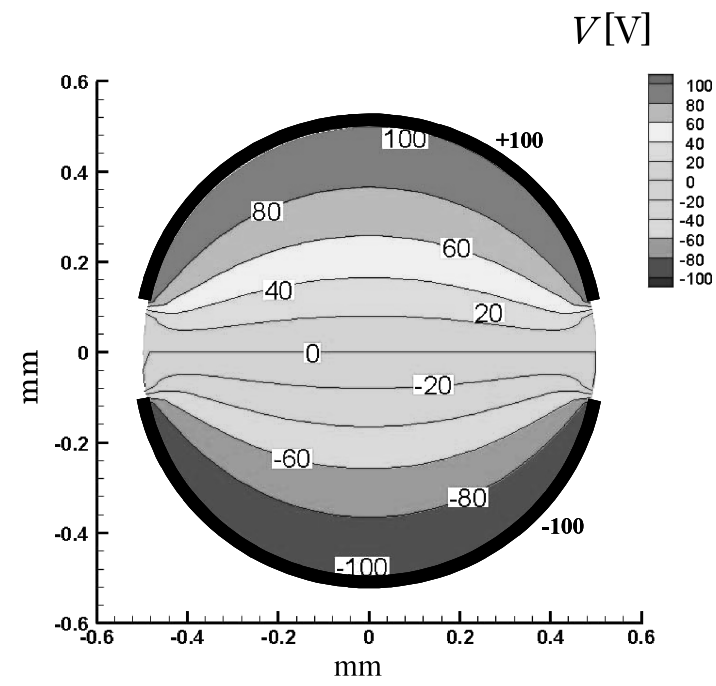

(a) Voltage

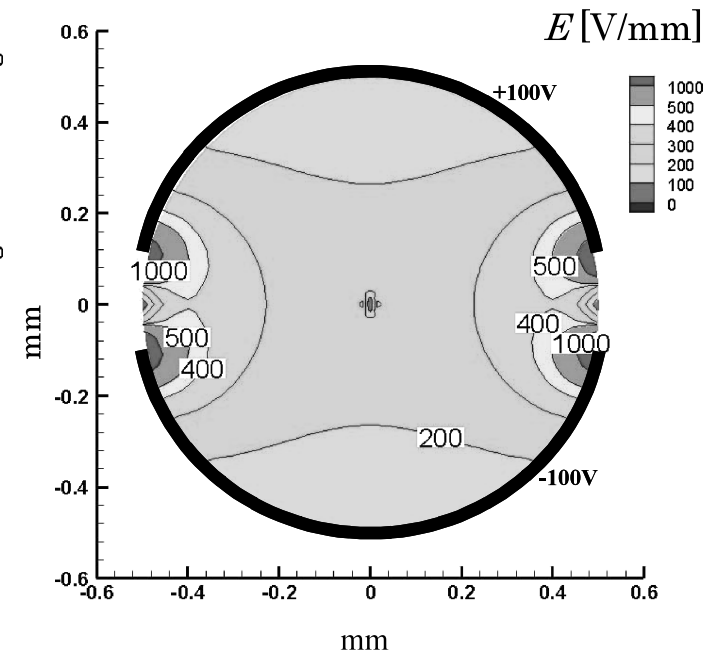

(b) Electric field intensity

Fig. 7 Electric field analysis for electrode B

when the electric field intensity is less than $1 \mathrm{kV} / \mathrm{mm}^{(2),(8)}$. The shear rates on the wall for the present circular-pipe electrode are almost ten times higher than those for the parallel-plate electrode assuming that the velocity distribution profile is parabolic. So the coefficient of the electric field intensity in Eq. (4) is assumed to be one-fifth (20\%) compared with that in the case of the parallel-pate electrode from the previous experimental results ${ }^{(9)}$, considering the difference in shear rate between the two electrodes.

\section{Results and Discussion}

The present calculated flow rate was compared with that of Hagen-Poiseuille's flow without voltages to confirm our calculation method. The error of the flow rate is less than $0.05 \%$ for $-1 \mathrm{kPa} / \mathrm{cm}$ of the pressure drop. The convergence condition of the present iteration method is $\max \left|\left(f^{n}-f^{n-1}\right) / f^{n}\right| \leq 5 \times 10^{-6}$, where $f$ is the voltage or the axial velocity component, $n$ is the calculation step and max stands for the maximum value in the flow field.

The calculated distributions of the voltage and the electric field intensity for electrodes A and B are shown in Figs. 6 and 7. A voltage of $200 \mathrm{~V}( \pm 100 \mathrm{~V})$ is applied to the electrodes. The electric field intensity is concentrated around the electrode strips, particularly near the edges of the electrode strips. The electric field intensity for electrode $\mathrm{B}$ is greater than that for electrode A near the center region $(r<0.25 \mathrm{~mm})$, and the region of electrode $\mathrm{A}$ in which $E>500 \mathrm{~V} / \mathrm{mm}$ near the wall is larger than that of electrode B. Thus, it is difficult to determine which elec- 


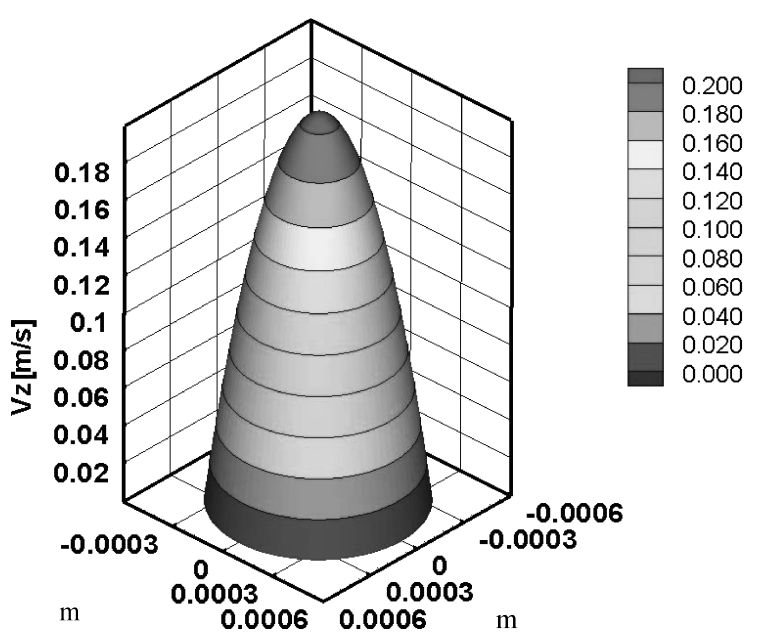

Fig. 8 Velocity distribution without voltage

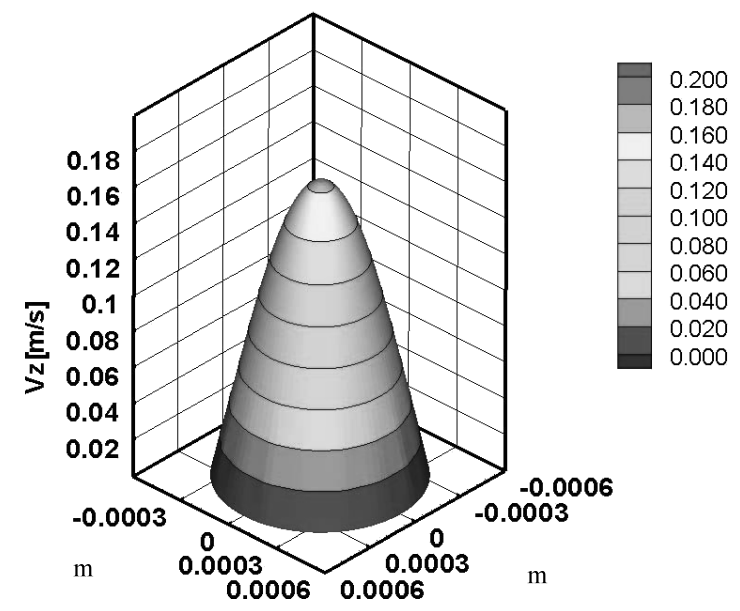

Fig. 9 Velocity distribution under $200 \mathrm{~V}$ for electrode A

trode has a stronger ER effect.

The examples of the velocity distribution calculated are shown in Figs. 8 and 9 for electrode A. The pressure drop is $-1 \mathrm{kPa} / \mathrm{cm}$ and the figures show the results for the situation without a field and for $200 \mathrm{~V}( \pm 100 \mathrm{~V})$ of the voltage. Of course the profile is parabolic without a voltage as shown in Fig. 8. The velocities decrease as a whole under application of the voltage in Fig. 9. The change of the electric field intensity in the peripheral direction near the wall can be seen in Fig. 6(b). However, the change of the velocity, which corresponds to the field, is almost not seen near the electrode strips in Fig. 9.

The calculated and experimental average pressure drops for the steady flow are shown in Figs. 10 and 11. In the present experiment the pressure drop of the circular pipe with the electrode strips was obtained by omitting the pressure drop without electrode strips from the measured values between the inlet and outlet of the total length of the pipe. The pressure drop without the electrode strips can be calculated theoretically using the assumption of the Hagen-Poiseuille's flow because the Reynolds number is
Electrode A

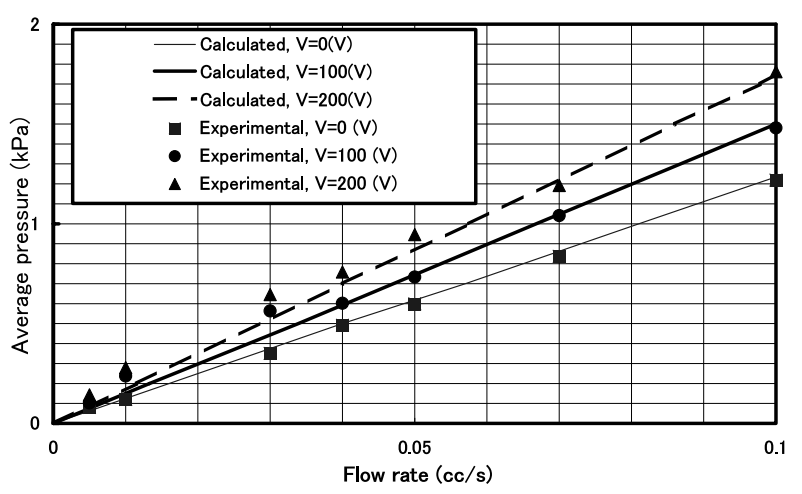

Fig. 10 Pressure drop for electrode A

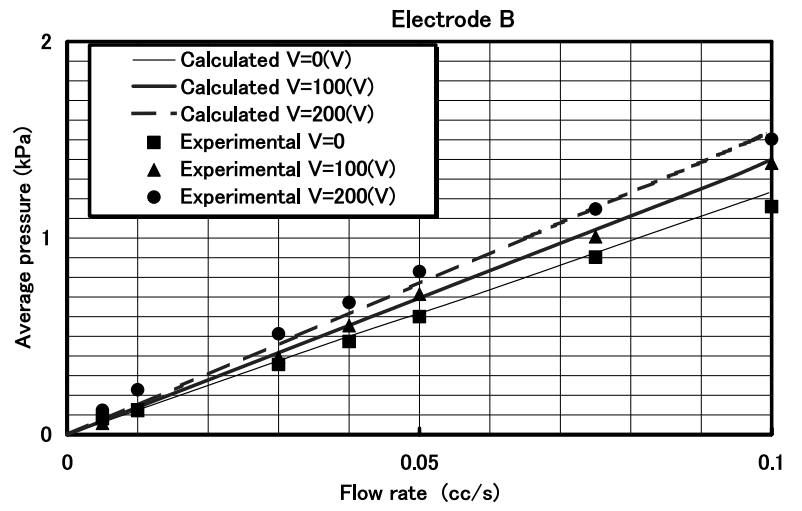

Fig. 11 Pressure drop for electrode B

less than 1.6. From Figs. 10 and 11, the pressure drops increase as the voltage or the volume flow rate increases. The pressure drop for electrode A increases by $40-50 \%$ under application of the voltage $(200 \mathrm{~V})$ compared with that with no field, while it is $30-40 \%$ for electrode B. Therefore, the ER effect for electrode A is stronger than that for electrode B in spite of having no field near the center region of the pipe. Thus, constructing many highintensity regions between the electrode strips near the inner surface is effective for obtaining a strong ER effect. The flow rates are obtained by integrating the velocity distribution calculated using Eq. (3) assuming that the viscosity is distributed in the flow field. The pressures estimated by our calculation are close to the experimental ones. The director of the liquid crystal is expected to be in the direction of the lines of the electric force, which are perpendicular to the equivoltage line shown in Figs. 6 (a) and 7 (a). It is found that the directors are almost in the radial direction in the fields with the high electric field intensity shown in Figs. 6 (b) and 7 (b). Thus, the viscosity can be predicted by the viscosity obtained experimentally using the parallel-plate electrode because the director in the radial direction for the circular-pipe electrode corresponds to that for the parallel-plate electrode under the application of the electric field.

As mentioned above, the present calculated results 
gap

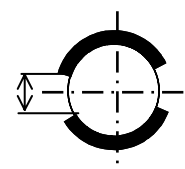

1 pair

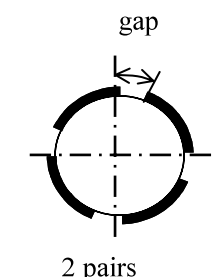

2 pairs

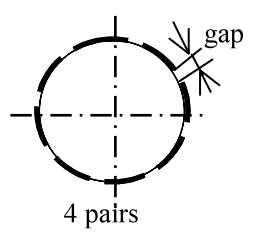

Fig. 12 Circular-pipe electrodes (internal diameter: $1 \mathrm{~mm}$ )
$\mathrm{V}=200 \mathrm{~V}$

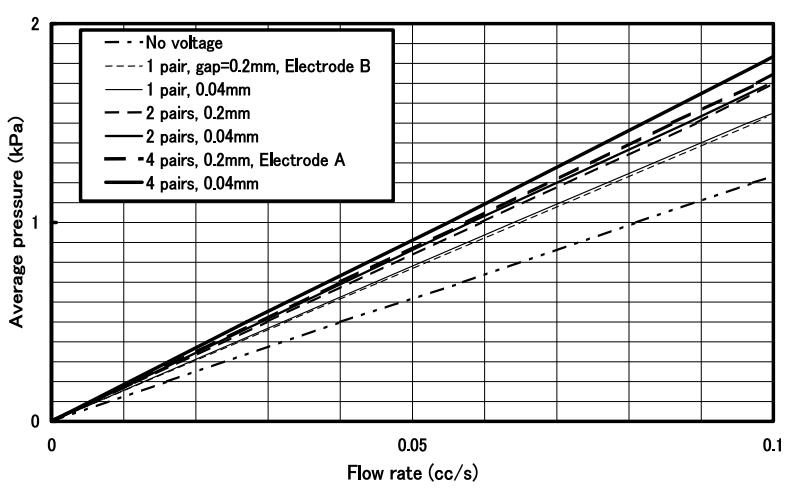

Fig. 13 Pressure drops for circular-pipe electrodes

agree with the experimental ones. Therefore, the effect of the number of electrode strips and the gaps between electrode strips on the pressure drop is examined by numerical calculation. As shown in Fig. 12, circular pipes with one pair, two pairs and four pairs of electrode strips are selected for the calculations. The gaps between the electrode strips are 0.2 and $0.04 \mathrm{~mm}$, and the six circularpipe electrodes are investigated. Equation (4) was used for the $0.04 \mathrm{~mm}$ gap, because the area for $E \geq 1 \mathrm{kV} / \mathrm{mm}$ is less than $3 \%$ of the cross-sectional area. The average pressure drops are shown under the application of $200 \mathrm{~V}$ in Fig. 13. The length of the electrodes is $10 \mathrm{~mm}$. The six calculated results are shown together with that for the case without any voltage. Electrode A has four pairs of electrode strips with $0.2 \mathrm{~mm}$ gaps and electrode $\mathrm{B}$ has a pair of electrode strips with the same gaps. At first it was found that increasing the number of electrode strips is useful to obtain a high pressure drop. Therefore we can see that increasing the number of electrode strips and the region of high electric field intensity near the circular-pipe wall is very effective in increasing the flow resistance and the pressure drop from the distribution of the electric field intensity shown in Figs. 6 (b) and 7 (b). Furthermore, decreasing the gap creates a high pressure drop for the same number of electrode strips. However, the constraints in the fabrication of the electrode should be considered.

The pressure drop and flow rate characteristics between the present circular electrodes and the two parallelplates electrode are compared on the basis of numerical simulations. The uniform electric field intensity is calculated by dividing the applied voltage by the distance be-

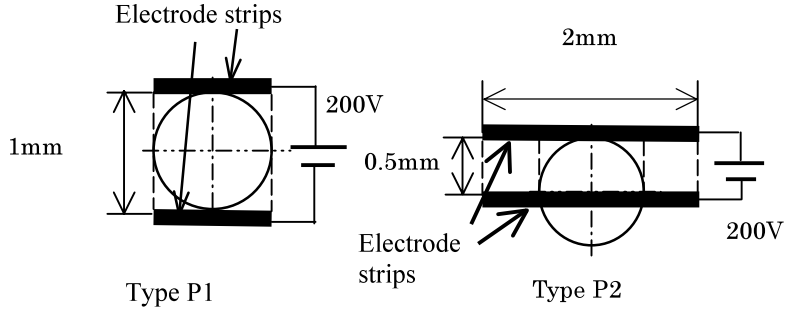

Fig. 14 Parallel-plate electrodes

tween electrode strips, and the characteristics are obtained from the exact solution of the Navier-Stokes equation for two parallel-plates electrode. Types P1 and P2 for the present parallel-plate electrodes are shown in Fig. 14. The gap and width of type P1 are both $1 \mathrm{~mm}$, while the gap is $0.5 \mathrm{~mm}$ and the width is $2 \mathrm{~mm}$ for type P2. The length of both electrodes is $10 \mathrm{~mm}$. The maximum shear rates on the wall of the parallel-plate electrode are $6001 / \mathrm{s}$ for type P1 and 1200 1/s for type P2, assuming that the velocity distribution profile is parabolic. That of the circular-pipe electrode is $1000 \mathrm{1} / \mathrm{s}$ and the comparison is done in almost the same order of the shear rate for Figs. 14 and 15. The comparison of the difference in pressure/flow rate characteristics between the two parallel-plates and the circularpipe electrodes is conducted under the condition that the area and the size of the cross section of the flow channel between two electrodes are not much different. The calculated results for one pair, two pairs and four pairs of electrode strips with $0.04 \mathrm{~mm}$ gaps in the case of the present electrode under the applied voltage of $200 \mathrm{~V}$ are shown in Fig. 15. The pressure for type P1 is about $25 \%$ of that for the circular pipe with four pairs of electrode strips. However, the difference of the pressure values between type P2 and the circular pipe with four pairs of electrode strips is within $10 \%$. From the present calculated results, the circular-pipe electrodes or the parallel-plate electrodes can be chosen according to their purpose. The comparison of the flow characteristics between the circular-pipe electrode and the parallel-plate electrodes is very useful for designing the micro-electrorheological valves, micromachines and other divices.

\section{Conclusions}

In this study, two types of circular-pipe electrodes with electrode strips on some parts of the inner surface of the pipe were developed and the pressure drops of a liquid crystal mixture flowing within the electrodes were measured at constant flow rates under applied electric fields. On the other hand, numerical simulations of the electric fields and fluid flow using the Navier-Stokes equation in the circular-pipe electrodes were conducted. It was assumed in the present analysis that the viscosity, which depends on the electric field intensity, is distributed in the flow fields. The relationship between the flow rate and the 


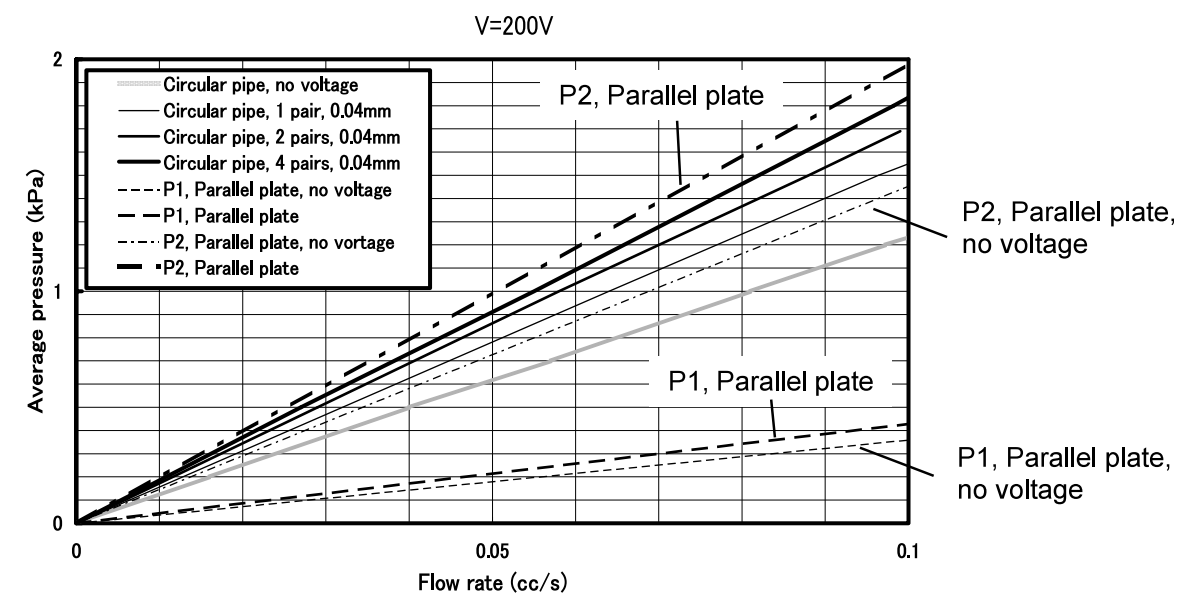

Fig. 15 Comparison between circular-pipe and parallel-plate electrodes

pressure were simulated numerically for various electric field intensities, which agree with the experimental results. Furthermore, the influence of both the number of electrode strips and the gaps between electrode strips on the pressure drop was investigated by the present simulations and the comparison of the flow characteristics between the present electrodes and two types of parallel-plate electrodes was conducted.

On the basis of the results obtained in the present investigation, the following conclusions can be drawn.

1. The pressure drops for the circular-pipe electrodes with one pair and with four pairs of electrode strips developed in our research increase by about $30-40 \%$ and $40-50 \%$ under the application of $200 \mathrm{~V}$, respectively, compared with those for no field.

2. The experimental relationship between the flow rate and the pressure drop for the circular-pipe electrodes can be estimated by the present theoretical approach, assuming that the viscosity is distributed in the flow field.

3. It is very useful to increase the number of electrode strips and use a small gap to obtain a high pressure drop.

4. The correspondence of the flow rate-pressure characteristics between the circular-pipe electrodes and the two types of parallel-plate electrodes is understood using the present simulation.

\section{Acknowledgments}

We thank Mr. Sumio Syuuto for his assistance in designing our experimental rig, MERCK Japan Co., Ltd. for supplying us the liquid crystal mixture, and Mr. Keisuke Nakayama and Mr. Tsuyoshi Mitani for their assistance.

\section{References}

( 1 ) Tsukiji, T. and Tanabe, S., ER Effect of Liquid Crys- tal Flowing between Two Parallel-Plate Electrodes, International Journal of Modern Physics B, Vol.16, No.17\&18 (2002), p.2569.

( 2 ) Tsukiji, T. and Mitani, T., Influence of Electric Fields on Pressure Drop of Liquid Crystal Mixture, 2002 ASME International Mechanical Engineering Congress and Exposition, (2002), CD-ROM, Vol.1, IMECE2002-32228.

( 3 ) Tsukiji, T. and Utashiro, T., Flow Characteristics of ER Fluids between Two Parallel-Plate Electrodes, Developments in Electrorheological Flows-1995, ASMEFED-Vol.235 (1995), p.37.

( 4 ) Nakano, M. and Yonekawa, T., Pressure Response of ER Fluid in a Piston Cylinder-ER Valve System, Electrorheological Fluids, Edited by Tao, R. and Roy, G.D., (1993), p.477, World Scientific.

( 5 ) Tsukiji, T. and Furuse, N., Flow Characteristics of ER Fluids between Concentric Cylinders, Developments in Electrorheological Flows and Measurement Uncertainty, ASME-FED-Vol.205, ASME-AMD-Vol.190 (1994), p.7.

( 6 ) Takesue, N., Furusho, J. and Sakaguchi, M., ER Effects of Liquid Crystalline Polymers on One-Sided Pattern Electrodes, Electro-Rheological Fluids and MagnetoRheological Suspensions, Edited by Bossis, G., (2001), p.174, World Scientific Publishing.

( 7 ) Tsukiji, T. and Mitani, T., Influence of Electric Fields on Flow of Liquid Crystal Mixture in a Circular Tube with Electrode Surface, Proceedings of FEDSM'03 4th ASME JSME Joint Fluids Engineering Conference, ASME, (2003), FEDSM2003-45047.

( 8 ) Tsukiji, T., Koyabu, E., Nakayama, K. and Mitani, T., Influence of Electric Fields on the Flow of a Liquid Crystal Mixture in a Circular Pipe with Electrode Surface, Trans. of the Japan Fluid Power System Society, (in Japanese), Vol.35, No.5 (2004), p.84.

( 9 ) Koyama, K., Application and Development of Electro Rheological Fluid, (1994), p.131, CMC. 